This item was submitted to Loughborough's Research Repository by the author.

Items in Figshare are protected by copyright, with all rights reserved, unless otherwise indicated.

\title{
Effects of random vibration in high-speed phase-shifting speckle pattern interferometry
}

\section{PLEASE CITE THE PUBLISHED VERSION}

PUBLISHER

(C) Optical Society of America

VERSION

AM (Accepted Manuscript)

\section{LICENCE}

CC BY-NC-ND 4.0

\section{REPOSITORY RECORD}

Ruiz, Pablo D., Jonathan M. Huntley, Yuji Shen, Charles R. Coggrave, and Guillermo H. Kaufmann. 2019. "Effects of Random Vibration in High-speed Phase-shifting Speckle Pattern Interferometry". figshare. https://hdl.handle.net/2134/5927. 
This item was submitted to Loughborough's Institutional Repository (https://dspace.lboro.ac.uk/) by the author and is made available under the following Creative Commons Licence conditions.

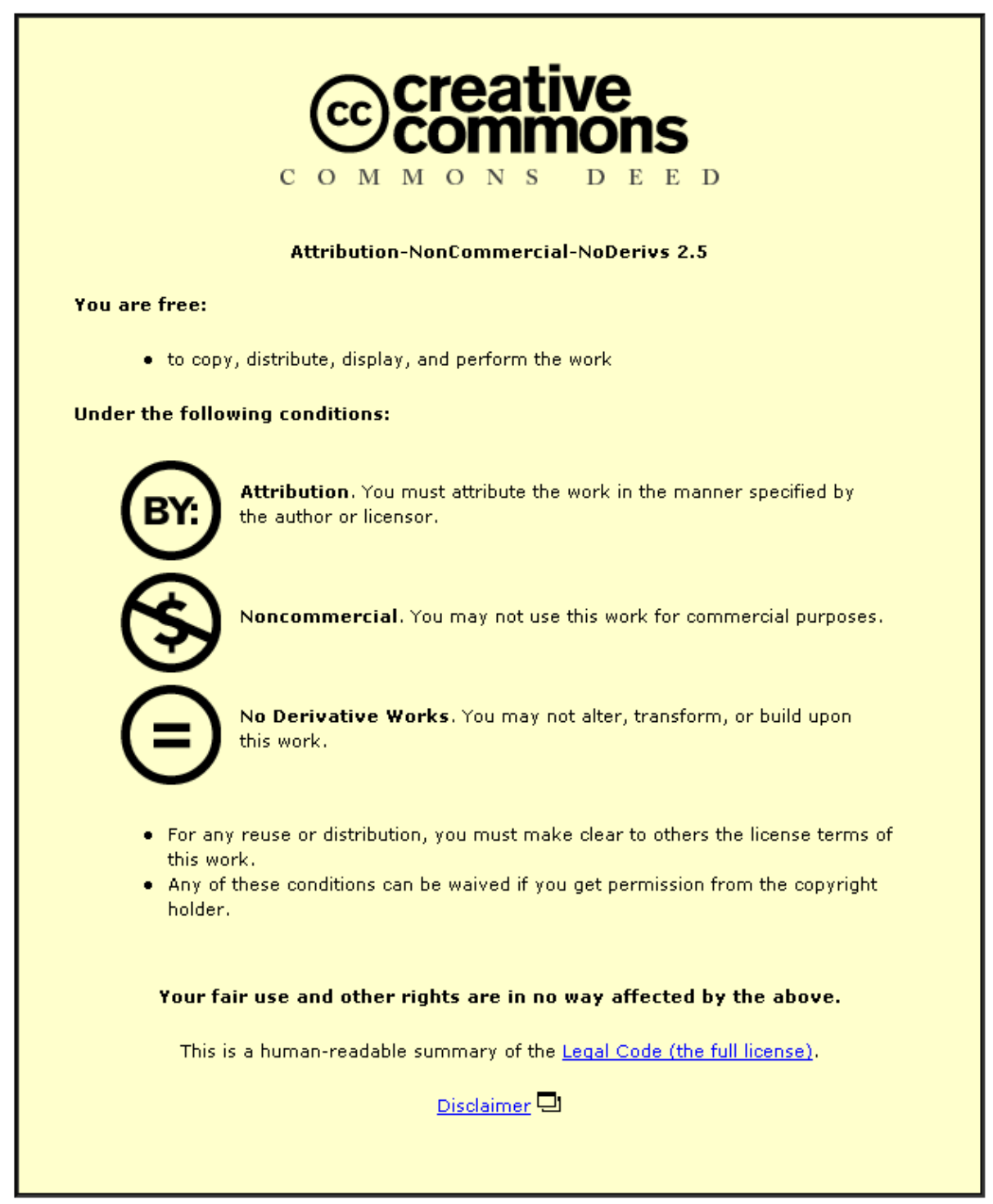

For the full text of this licence, please go to: http://creativecommons.org/licenses/by-nc-nd/2.5/ 


\title{
Effects of random vibration in high-speed phase-shifting speckle pattern interferometry
}

\author{
Pablo D. Ruiz \\ Instituto de Física Rosario, 2000 Rosario, Argentina \\ e-mail: ruiz@ifir.ifir.edu.ar
}

\section{Jonathan M. Huntley}

Department of Mechanical Engineering, Loughborough University, Loughborough, Leics. LE11 3TU, UK

e-mail: J.M.Huntley@1boro.ac.uk

\section{Yuji Shen}

Department of Mechanical Engineering, Loughborough University, Loughborough, Leics. LE11 3TU, UK

e-mail: Y.Shen@lboro.ac.uk

\section{Russell Coggrave}

Department of Mechanical Engineering, Loughborough University, Loughborough, Leics. LE11 3TU, UK

e-mail: C.R.Coggrave@lboro.ac.uk

\section{Guillermo H. Kaufmann}

Instituto de Física Rosario and Departamento de Física, Facultad de Ciencias Exactas, Ingeniería y Agrimensura, Universidad Nacional de Rosario, 2000 Rosario, Argentina

e-mail: guille@ifir.ifir.edu.ar 


\begin{abstract}
The influence of random vibrations on a dynamic phase shifting speckle pattern interferometer, in which phase difference evaluation is performed using temporal phase shifting and temporal phase unwrapping, is investigated by means of experiments and numerical simulations. A well-defined velocity spectral density function, typical of the spectra found under non-vibration-isolated conditions, is used throughout. Five phase-shifting formulae are studied, with camera framing rates $(1,2$ and $4 \mathrm{kHz}$ ) typical of current dynamic speckle pattern interferometers. Two main aspects were evaluated: firstly the unwrapping reliability, and secondly the noise induced in the phase maps by the vibration. The former was found to be a significant constraint, even for peak velocities well below the Nyquist velocity limit of the interferometer, and is therefore likely to be more important than the latter in many applications. Three analytical criteria for determining the expected unwrapping success rate are proposed and their predictions compared with the measured values. It is demonstrated that shorter sampling windows and higher framing rates are preferred in order to increase the unwrapping success rate, but that longer windows reduce the root mean square error in the phase change maps due to the vibration.
\end{abstract}

OCIS codes: $120.6160,120.5050,120.2650,120.7280,120.4290$. 


\section{Introduction}

Dynamic electronic speckle pattern interferometry (ESPI) constitutes a recent trend among coherent optical techniques for measuring dynamic deformation fields in solid mechanics. ${ }^{1-4}$ The most powerful dynamic speckle interferometers are those based on a high-speed image sensor which incorporate a phase modulator in the object or reference beam. Temporal phase shifting at the framing rate of the camera allows wrapped phase maps to be calculated at the time of each frame of the image sequence. These phase maps can then be unwrapped as a function of time to provide the end user with a movie of the absolute object deformation state, even in the presence of global discontinuities such as specimen boundaries and cracks. ${ }^{5}$ The main strengths of this technique, common to the vast majority of speckle interferometry methods, are its non-contacting nature, high sensitivity, and minimum surface preparation requirements. ${ }^{6}$

Unfortunately, all PS algorithms are susceptible to some extent to systematic errors such as the presence of higher harmonics in the temporal intensity signal, phase shifter miscalibration or nonlinear response of the photodetectors. ${ }^{7}$ Vibration is also a significant source of phase error when a PS interferometer is employed away from a vibration-isolated table. The effect of vibration on the unwrapped phase depends mainly on the spectral content of the vibration, its amplitude, the particular PS algorithm used and the object phase itself. The object phase dependence is the most damaging, since the phase error appears as a ripple with twice the spatial frequency of the original fringe pattern (in smooth wavefront interferometers) or as a spatially distributed random noise (in speckle interferometers).

Useful insight into the sensitivity of different PS algorithms to vibration was given by de Groot and Deck, ${ }^{8,9}$ who calculated the rms ripple phase error by 
evaluating the propagation of intensity errors through the PS algorithm. They worked with standard smooth-wavefront interferometry rather than dynamic speckle interferometry and studied the case of single frequency vibration. It was found that the largest ripple phase error is produced at a vibration frequency equal to twice the intensity-modulation frequency produced by the phase shifting device. More recently, the sensitivity of a dynamic PS interferometry system to single frequency vibration was numerically analyzed by the present authors. ${ }^{10}$ It was shown that the rms phase error frequency response depends mainly on the length and shape of the sampling window from which the PS algorithm coefficients are evaluated, and also on the framing rate of the camera.

In the light of these works, a further step is the evaluation of the sensitivity of the dynamic ESPI system to random vibration, which is a common noise source in real environments. A particular case occurs when the random vibration can be considered as a rigid body motion added to the test object. Such a situation arises when carrying out relatively low strain-rate tests on different materials, using for example, loading by vacuum or by tensile test machine. In these cases, piston-like random vibration can lead to spatial dispersion of the phase change values and even to severe phase evaluation failure. Therefore, it is important to know in which circumstances the system will be able to track the phase when a random vibration is present and to measure the phase dispersion between pixels.

In order to evaluate the dynamic high-speed speckle interferometer under these conditions, we designed an experiment in which the target was driven with synthesized random displacements. At the same time, numerical simulations were carried out to gain insight into the effects of the random vibration. Advantages of the numerical simulation are that it allows the isolation of certain noise sources that are 
added in the experiment, and that the parameters of interest (vibration rms amplitude and spectral content, and camera framing rate) can be studied over a wider range than that allowed by the experimental equipment. Furthermore, statistical averages such as the unwrapping success rate require large numbers of datasets which would take prohibitive amounts of time if carried out experimentally.

In Section 3 we describe the simulation and introduce two parameters to assess firstly the probability of successful phase evaluation and secondly the average rms phase error due to the vibration alone. The performance of several PS algorithms was studied when random vibration of known spectral content and rms amplitude are present. Discussion and conclusions on the best choice of PS algorithm and framing rate under given circumstances are finally given in Sections 4 and 5 , respectively.

\section{Experimental}

\subsection{Dynamic phase-shifting interferometer}

Figure 1 shows a schematic view of the out-of-plane high-speed ESPI system. In the optical setup, the beam of a frequency-doubled continuous-wave $\mathrm{Nd}$ :YAG laser (wavelength $\lambda=532 \mathrm{~nm}$ ) is divided into object and reference arms by a 90:10 beam splitter. The reference beam is passed through a Pockels cell and recombined with the speckle pattern formed by the scattered object beam using a second 90:10 beam splitter in front of the camera objective. The Pockels cell is driven by a staircase waveform generator which produces one out of a set of four equally-spaced voltage levels, which are clocked cyclically in response to the rising edges of a TTL $1 \mathrm{kHz}$ square wave input signal. A function generator is used to produce the square wave, which also drives the high-speed video camera, and 
therefore acts as the master clock for the whole experiment. A detailed description of the speckle interferometry system and of the calibration procedure can be found in Ref. 2.

The test object consisted of an aluminum disk attached to a piezo-electric translator (PZT), which was submitted to a high voltage random signal to move it rigidly back and forth. The method of generating the signal is described in Section 2.2. The gain of the high voltage amplifier was set to produce random displacements of the disk with the following rms amplitudes: $\lambda / 32, \lambda / 16, \lambda / 8, \lambda / 4$ and $\lambda / 2$. Higher rms displacement amplitudes were outside the voltage range of the PZT. The PZT was tightly clamped to a heavy steel block, which was in turn screwed to the optical table in order to reduce the excitation of structural resonant frequencies in the support. A trigger signal was used to start the digital data transfer and also the phase stepping and the frame recording by the Pockels cell and the camera, respectively. Approximately 1.6 seconds of signal can be recorded at full frame resolution. The recorded frames were then downloaded to the Sun SPARCstation through a GPIB interface. After that, the phase change values were evaluated and unwrapped using the modified algorithm presented in Ref. 2 (Eqs. (15) - (17)), which allowed spatial speckle averaging to be carried out over clusters of $3 \times 3$ pixels.

\subsection{Generation of pseudo-random vibrations}

The method used to generate the pseudo-random vibrations signal was common to both the experimental and the numerical simulation sections of this paper. In both cases, the required signal is for target displacement, whereas normally the spectral content of vibration is specified in terms of target velocity. Design criteria for metrology laboratories are often based on either peak velocity spectra or probability density spectra for velocity. These are typically specified to be 
flat from dc up to a corner frequency, $f_{0}$, with a high frequency roll-off. ${ }^{11,12}$ This approach was adopted in the present study by specifying a spectral density function for velocity, $S(f)$ as follows:

$$
S(f)=\left\{\begin{array}{cr}
S_{0} & 0<f \leq f_{0} \\
\frac{S_{0} f_{0}}{f} & f_{0}<f
\end{array}\right.
$$

where $S_{0}$ is the spectral density for the flat portion of the spectrum (with units of $\mu \mathrm{m}^{2}$ $\mathrm{s}^{-1}$ ). This function has a $20 \mathrm{~dB}$ per decade roll-off above the corner frequency $f_{0}$, the value of which was fixed at $50 \mathrm{~Hz}$. The severity of the vibration was controlled by varying the single parameter $S_{0}$.

The desired spectral content was achieved by frequency-domain filtering a one-dimensional array of independent random numbers from a normal distribution $\mathrm{N}(0,1)$, i.e. with zero mean and unit variance. A $20 \mathrm{~dB} / \mathrm{dec}$ rolloff filter was applied to its amplitude spectrum from DC to the corner frequency $f_{0}$, and another $20 \mathrm{~dB} / \mathrm{dec}$ rolloff above $f_{0}$. The real part of the inverse Fourier transform of the filtered spectrum, after suitable scaling, then specified the sample displacement time history $u_{\mathrm{z}}(\tau)$. Figure 2 is an example of an average of 100 independent velocity spectra created in this way with a scaling chosen such that the rms displacement amplitude was $\sigma_{z}=\lambda / 2$. The spectrum is seen to follow the form specified by Eqn. (1), with a mean spectral density at the plateau $S_{0}=0.51$. The particular $S_{0}$ value of the velocity signal used in the experiments was 0.49 for $\sigma_{z}=\lambda / 2$.

In order to drive the PZT, the data were scaled from 0 to $10 \mathrm{~V}$. They were written to a Digital-to-Analogue converter by a second computer, which had a realtime operating system (LynxOS) in order to guarantee a constant read-out rate. The chosen rate was 1220 samples per second, giving a total of 2.5 seconds of analog 
random noise. A signal conditioner was used to filter out high frequencies due to the stepped digital signal and finally a high voltage amplifier was used to drive the PZT.

\subsection{Example of phase dispersion}

Figure 3 shows a short sample of the complete time history of the unwrapped phase for seven different $3 \times 3$ pixel clusters, evaluated with the Carré algorithm from experimental data with a rms displacement vibration amplitude of $\lambda / 4$. The bold line corresponds to the average phase of the different clusters. The dispersion of the phase from the different clusters can be clearly appreciated. This dispersion manifests itself in the phase images as spatial fluctuations and therefore introduces unwanted noise into the final displacement maps. It is likely that these phase errors result from a combination of several sources: intensity errors, quantization errors, speckle decorrelation, nonlinearities of the image sensor and the phase stepping device, as well as the vibration itself. ${ }^{13,14}$

\section{Numerical Simulation}

Simulations were carried out using the model from Ref. 2, but with the sinusoidal vibration replaced by the random vibration signals generated as described in Section 2.2. In brief, $\tau_{j}=j \delta \tau$ defines a discrete time axis with $j=0,1,2, \ldots, J-1$, where $\delta \tau$ is a time increment (in units of s) and $J$ is the total number of points along the time axis. In the presence of a vibration, the light intensity at a given camera pixel and time $\tau_{j}$ is given by the usual phase-shifting interferometry equation:

$$
I\left(\tau_{j}\right)=I_{0}\left\{1+V \cos \left\lfloor\Phi\left(\tau_{j}\right)+\Phi_{v}\left(\tau_{j}\right)+\phi\left(\tau_{j}\right)\right\rfloor\right\},
$$

where $I_{0}$ is the local average intensity; $V$ is the speckle visibility; $\Phi$ is the object phase that is related to the displacement field to be measured and which includes a speckle random phase; $\Phi_{v}$ is the optical phase introduced by the random vibration; 
and $\phi\left(\tau_{j}\right)$ is the phase shift for time $\tau_{j}$. In this simulation $\Phi$ contains only the speckle phase, as the disk is not submitted to a deformation.

The pseudo-random vibration phase $\Phi_{v}$ is taken from the same data file which was used to generate the experimental random vibrations described in Section 2. After matching the sampling points of the data file to those of the simulation by means of a spline interpolation, the phase amplitude of the signal is set to a particular rms value $\sigma_{\phi}$. For an out-of-plane interferometer, $\sigma_{\phi}$ is related to the rms normal displacement $\sigma_{z}$ of the sample by:

$$
\sigma_{\phi}=\frac{4 \pi}{\lambda} \sigma_{z}
$$

The phase-stepping function $\phi\left(\tau_{j}\right)$ is as specified in Ref. 2 , and is designed to give phase steps of $\Delta \phi=\pi / 2$ every $p$ time steps. The time-integration effect of the image sensor is accomplished by adding the resulting calculated intensity values within each frame integrating period $T_{f}=p \delta \tau$. The result is a sequence of intensity values, $\bar{I}(t), t=0,1,2, \ldots, N_{t}-1$ where $N_{\mathrm{t}}$ is the total number of simulated frames.

\section{1 Phase evaluation}

A wrapped estimator $\hat{\Phi}_{w}$ of the addition of the object phase $\Phi$ and the vibration phase $\Phi_{v}$ can be obtained as:

$$
\hat{\Phi}_{w}(t)=\tan ^{-1}\left(\frac{N(t)}{D(t)}\right),
$$

where

$$
N(t)=\operatorname{Im}[z(t)], \quad D(t)=\operatorname{Re}[z(t)]
$$

and

$$
z(t)=\left[\sum_{t^{\prime}=0}^{M-1} a\left(t^{\prime}\right) \bar{I}\left(t+t^{\prime}\right)+i \sum_{t^{\prime}=0}^{M-1} b\left(t^{\prime}\right) \bar{I}\left(t+t^{\prime}\right)\right] \exp (-i \Delta \phi t)
$$


where the coefficients $a(t)$ and $b(t)$ depend on the particular PS algorithm used.

Defining:

$$
a(t)+i b(t)=w(t) e^{-i \Delta \phi t}, \quad t=0,1,2, \ldots, M-1
$$

it can be seen that the term between the square brackets in Eq. (6) is equivalent to the Fourier transform of a windowed intensity signal evaluated at the modulation frequency $k_{t}=1:^{15,16}$

$$
\tilde{I}\left(k_{t}, t\right)=\sum_{t^{\prime}=0}^{M-1} \bar{I}\left(t+t^{\prime}\right) w\left(t^{\prime}\right) \exp \left(-2 \pi i k_{t} t^{\prime} / N\right) .
$$

The window coefficients $w(t)$ can be easily calculated from the specified sampling coefficients $a(t)$ and $b(t)$ (or vice versa) using Eq. (7). In this paper, the following PS algorithms were evaluated:

\section{a) Carré algorithm}

This algorithm uses the phase shift as another unknown variable. If it is assumed that the phase shifts are identical, then there are four unknowns: $I_{0}, V, \Phi_{v}$ and $\Delta \phi$, and therefore four frames are enough to evaluate $\Phi_{v}$. This algorithm cannot be expressed in terms of Eqn. (7) since the numerator is a non-linear combination of measured intensities:

$$
\begin{aligned}
& \hat{\Phi}_{w}(t+3 / 2)= \\
& \tan ^{-1}\left\{\frac{S \sqrt{[\bar{I}(t)-\bar{I}(t+3)+\bar{I}(t+1)-\bar{I}(t+2)]\{3[\bar{I}(t+1)-\bar{I}(t+2)]-\bar{I}(t)+\bar{I}(t+3)}\}}{\bar{I}(t+1)+\bar{I}(t+2)-\bar{I}(t)-\bar{I}(t+3)}\right\}
\end{aligned}
$$

where $S=\operatorname{sign}[\bar{I}(t+1)-\bar{I}(t+2)]$ and where $\operatorname{sign}(x)=1$ for $x>0$ and -1 otherwise.

The sign function gives the correct sign to the numerator, allowing a full fourquadrant phase value to be obtained by means of the atan 2 function. 


\section{b) Hanning window algorithms}

In this case, the sampling coefficients were evaluated through Eq. (7) using the well known raised cosine or Hanning window:

$$
w(t)=\frac{1}{2}+\frac{1}{2} \cos \left\{\frac{2 \pi}{M}\left[t-\left(\frac{M-1}{2}\right)\right]\right\} \quad t=0,1, \ldots, M-2 .
$$

Note that in this definition, the window width is $M$ while $M-1$ frames are used. $^{15}$

\section{2. Temporal phase unwrapping}

Temporal phase unwrapping refers to the process of adding integral multiples of $2 \pi$ to the wrapped phase estimator $\hat{\Phi}_{w}(t)$ such that the magnitude of the phase change between two successive unwrapped phase values $\hat{\Phi}_{u}(t)$ lies in the range $[-\pi$, $\pi)$. Temporal unwrapping is performed by determining the number $d(t)$ of $2 \pi$ phase jumps between two successive wrapped phase values by means of:

$$
d(t)=\operatorname{NINT}\left\{\left[\hat{\Phi}_{w}(t)-\hat{\Phi}_{w}(t-1)\right] / 2 \pi\right\}, \quad t=1,2, \ldots, s
$$

where $s=N_{\mathrm{t}}-M$ and NINT denotes round to the nearest integer.

The total number of phase jumps $v(t)$ is calculated as:

$$
v(t)=\sum_{t^{\prime}=1}^{t} d\left(t^{\prime}\right), \quad t=1,2, \ldots, s
$$

and the unwrapped phase change value is obtained as:

$$
\Delta \hat{\Phi}_{u}(t, 0)=\hat{\Phi}_{w}(t)-\hat{\Phi}_{w}(0)-2 \pi v(t), t=1,2, \ldots, s
$$

with $\Delta \hat{\Phi}_{u}(0,0)=0$. The wrapped phase subtraction evaluated in Eq. (13) eliminates the random speckle phase, which is present in the wrapped phase values $\hat{\Phi}_{w}(t)$. 


\section{3. Unwrapping success rate and average rms phase error}

It is useful to assess the unwrapping success rate of a given PS algorithm when the phase of a vibrating surface is determined. This parameter is defined as the ratio of the successfully unwrapped signals to the total number of unwrapped ones. ${ }^{17}$ The unwrapping of a wrapped phase time sequence of given length is assumed to be correct if the unwrapped phase agrees with the corresponding input phase to within $\pm \pi$ for the last frame of the sequence. Even though this condition may be fulfilled for the last frame, large phase gradients can cause the unwrapped and the input phases to split out and to match again by chance during some time within the sequence. Though this case should not be considered strictly as a successful unwrapping, it is quite rare, at least for the cases of interest where the probability of a successful unwrapping is close to 1 . The unwrapping success rate does not refer to the unwrapping process alone, but mainly to the phase evaluation procedure. The numerical simulation allows us to evaluate this parameter for several conditions that cannot be carried out experimentally and also to associate the cause of failure to the vibration itself and not to other sources of error.

In a speckle interferometer, the phase change estimator $\Delta \hat{\Phi}_{u}$ depends on a reference speckle phase $\hat{\Phi}_{w}$ that varies from point to point in space and that is uniformly distributed over the range $[-\pi, \pi)$. This spatial variation makes the phase change values vary from one pixel to another. In the simulations, therefore, $L$ object phase values $\Phi_{l}$ have been chosen equally spaced over the range $[-\pi, \pi)$ and the index $l$ was used to designate them. Moreover, any frame $t_{j}$ can be considered as the reference frame for the remaining ones. Therefore, the rms phase change error $\sigma\left(t, t_{j}\right)$ 
for a given time $t$ and reference frame $t_{j}$, has to be averaged over all possible object phase values $\Phi_{l}$. This can be written as:

$$
\sigma\left(t, t_{j}\right)=\left\{\frac{1}{L} \sum_{=0}^{L-1}\left[\Delta \hat{\Phi}_{u}\left(t, t_{j}, l\right)-\left\langle\Delta \hat{\Phi}_{u}\left(t, t_{j}\right)\right\rangle^{2}\right\}^{1 / 2},\right.
$$

with

$$
\left\langle\Delta \hat{\Phi}_{u}\left(t, t_{j}\right)\right\rangle=\frac{1}{L} \sum_{l=0}^{L-1} \Delta \hat{\Phi}_{u}\left(t, t_{j}, l\right) .
$$

An example of $\left\langle\Delta \hat{\Phi}_{u}\left(t, t_{j}\right)\right\rangle$ from one of the experiments is shown in Fig. 3 as a bold line, whilst $\sigma\left(t, t_{j}\right)$ measures the dispersion of the phase change obtained for different pixels. $\sigma\left(t, t_{j}\right)$ varies rapidly in time and also depends on the reference frame chosen. After averaging over these indexes, we obtain:

$$
\bar{\sigma}=\frac{1}{s \cdot s_{j}} \sum_{t=1}^{s} \sum_{t_{j}=1}^{s_{j}} \sigma\left(t, t_{j}\right)
$$

where $s_{j}$ is the number of reference frames considered and $s$ has been already defined above. In this way, $\bar{\sigma}$ represents an average rms measure of the phase change dispersion between pixels. This definition of phase error differs from that given in Ref. 2, and is more appropriate for the case where one is concerned about vibrationinduced spatial fluctuations in the evaluated phase rather than about the rigid body translation of the whole sample.

\section{Numerical and experimental results and discussion}

\subsection{Unwrapping success rate}

The unwrapping success rate of different PS formulae was evaluated numerically by use of the simulation described above. The dynamic PS speckle interferometer used for the experiments described in Section 3 was modeled as if it were operating in the presence of random vibration with rms phase amplitudes $\sigma_{\phi}=$ $\pi / 2, \pi, 3 \pi / 2,2 \pi$ and $5 \pi / 2$ radians, by setting: $N_{\mathrm{t}}=1000,2000$ and 4000 frames; $I_{0}=$ 
$0.5 ; V=1 ; p=11 ; T_{f}=1 / 1000,1 / 2000$ and $1 / 4000$ seconds $; \delta \tau=T_{f} / p$ seconds $; \Delta \phi=$ $\pi / 2 \operatorname{rad}$ and $L=15$. The coefficients $a(t), b(t), w(t)$ and $M$ were imposed by the particular PS algorithm used.

Table 1 shows the unwrapping success rate for the different PS algorithms tested when the phase of a random vibration was evaluated for different rms amplitudes and framing rates. Each value was obtained by evaluating 100 onesecond duration random signals, all of them generated as described in Section 2, with a spectral content similar to the one shown in Fig. 2. It is seen that the success rate decreases as the rms surface displacement increases, and also when the PS algorithm window size increases. Combinations having a given product of framing rate and number of frames $M$ in the PS algorithm have approximately the same success rate, suggesting that it is the time duration of the PS window, $T_{w}$, which controls the unwrapping reliability.

Some insight into this behaviour is provided by plotting the windowed Fourier transform (WFT) of the intensity signal. ${ }^{3}$ Figure 4 (a) shows the velocity of the surface for a rms phase amplitude of $\sigma_{\phi}=\pi / 2$ or, equivalently, $\sigma_{z}=\lambda / 8$. Figures 4(b) and 4(c) show the modulus of $\tilde{I}\left(k_{t}, t\right)$ calculated by Eq. (8) when a Hanning window was used for $M=32$ and $M=64$, respectively. A vertical slice through the images shown in Figs. 4(b) and 4(c) at a point $t$ along the horizontal axis corresponds to the magnitude of the Fourier transform of the $M$ intensity values starting at frame $t$. Low frequency variations in the surface velocity manifest themselves as shifts in the peak position of the modulus function away from the line $k_{t}=1$ (the carrier frequency). High frequency variations result in broadening and splitting of the signal peak. Both effects are visible in Figs. 5(b) and 5(c), where the rms vibration amplitude is $\sigma_{z}=\lambda / 2$. The phase extraction formula described by Eq. (4) evaluates 
the phase at a fixed frequency $k_{t}=1$; carrying out the phase unwrapping along this line will then result in the accumulation of a $\pm 2 \pi$ phase unwrapping error if a zeromodulus hole appears between the line and the signal peak. ${ }^{1}$ In the remainder of this sub-section we investigate three simple criteria based on these observations, with the aim of predicting an expected unwrapping success rate for a given spectral content of the vibration signal.

Criterion 1: Nyquist velocity exceeded by at least one random noise peak.

The Nyquist velocity is the main limiting factor for sinusoidal excitation ${ }^{10}$ and this is perhaps therefore the most obvious criterion.

The expected number of velocity peaks in unit time, whose magnitude is greater than a given velocity value $v_{n}$, can be written: ${ }^{18}$

$$
\mu=2\left(\frac{\int_{f_{1}}^{f_{2}} f^{2} S(f) d f}{\int_{f_{1}}^{f_{2}} S(f) d f}\right)^{1 / 2} \exp \left(-\frac{v_{n}^{2}}{2 \int_{f_{1}}^{f_{2}} S(f) d f}\right)
$$

where $f_{1}$ and $f_{2}$ are respectively the lower and upper frequencies present in the vibration spectrum. Equation (17) differs from that specified in Ref. 18 by a factor of 2 since negative velocity peaks are as damaging as positive velocity peaks. The probability of a given number of peaks in time $\tau$ follows the Poisson distribution. In particular, the probability of zero peaks, which in this model can be equated to the unwrapping success rate, is given by

$$
P(0)=\exp (-\mu \tau)
$$

Substituting the values $f_{1}=0, f_{2}=610 \mathrm{~Hz}$ (the Nyquist frequency limit for the D-A converter), the Nyquist velocity limit of $\mathrm{v}_{\mathrm{n}}= \pm 66.5 \mathrm{~mm} \mathrm{~s}^{-1}$ (for a $1 \mathrm{kHz}$ framing rate), and the expression for spectral density given by Eqn. (1) with $S_{0}=0.51 \mu \mathrm{m} \mathrm{s}^{-2}$, we find a value $\mu=3 \times 10^{-9} \mathrm{~s}^{-1}$. This would imply an unwrapping failure about once every 10 years, as opposed to the much more frequent failures observed in practice. 
Furthermore, such a criterion predicts the unwrapping success rate to be independent of PS window size, in contrast to the results shown in Table 1. For both these reasons, such a criterion has to be regarded as inappropriate when dealing with random vibrations, at least for this particular combination of spectral density and camera framing rate.

Criterion 2: Signal peak shifted by the signal peak width, due to low-frequency vibration.

The width of the signal peak (from maximum to first zero) is obtained from the Fourier transform of the window function as $\Delta k_{\mathrm{t}}=8 / M$ for the case of a Hanning window. ${ }^{10}$ The scaling between the shift in the signal peak, $\delta k_{\mathrm{t}}$, and out-of-plane velocity component $v_{\mathrm{z}}$ is as follows:

$$
\delta k_{t}=8 T_{f} v_{z} / \lambda
$$

where $T_{\mathrm{f}}$ is the camera interframe time. Unwrapping failure can be expected when $\delta k_{\mathrm{t}} \geq \Delta k_{\mathrm{t}}$. The distinction between low and high frequency vibration is somewhat arbitrary, but since the relevant timescale is the PS window duration $T_{\mathrm{w}}$, an appropriate order-of-magnitude cut-off frequency is $f_{\mathrm{w}}=2 / T_{\mathrm{w}}$ (for Hanning windows). The combination of Eqns (17) (with limits $f_{1}=0$ and $f_{2}=f_{\mathrm{w}}$ ), (18) and (19) allows the unwrapping success rate to be calculated according to this criterion. However, the results are again found to be over-optimistic.

Criterion 3: Ridge splitting due to high-frequency vibration. Splitting of the ridge (seen for example in Fig. 5(c)) occurs due to significant changes in velocity within the timescale of the window duration, $T_{\mathrm{w}}$. It is therefore due principally to the higher frequency components of the velocity spectrum. Unlike the two previous criteria there is no obvious analytical method of predicting the onset of splitting. We therefore make the assumption that it will occur if a velocity 
peak, due to the frequency components of the spectrum above $f_{\mathrm{w}}$, exceeds some threshold value $v_{\mathrm{T}}$. We chose $v_{\mathrm{T}}=22 \mu \mathrm{m} \mathrm{s}^{-1}$ (i.e., one third of the Nyquist velocity), which minimises the rms error between the simulation and analytical results of the success rate.

Table 2 shows the predicted unwrapping success rates based on this criterion for the case $v_{\mathrm{T}}=22 \mu \mathrm{m} \mathrm{s}^{-1}$, obtained by the use of Eqns. (17)-(19) with limits $f_{1}=f_{\mathrm{w}}$ and $f_{2}$ $=610 \mathrm{~Hz}$. The results show the correct trend: unwrapping reliability decreases with increasing window size, a result which can be explained in simple terms as being due to the inclusion of a progressively greater fraction of the spectrum $S(f)$. In view of the crude nature of the approximation used, the agreement with the numerical results is not too bad, although the changes in unwrapping reliability take place over a narrower range of vibration amplitudes than is observed in the simulations. The overly pessimistic prediction at high excitation levels could be explained in part by cases where unwrapping errors of opposite sign cancel out, thereby counting as an unwrapping success in the numerical simulations but as a failure in this empirical model.

The third criterion seems the most appropriate for the current case and suggests that signal peak splitting may be the most important source of unwrapping error when the ratio of camera framing rate to corner frequency $f_{0}$, takes the current value of 20:1. However, it is to be expected that this ratio can be increased as cameras with higher framing rates become available; splitting of the signal peak would then be expected to become less severe and the second criterion could become the dominant factor in determining unwrapping success rate. An adaptive PS algorithm capable of following the ridge would then show significant benefits over the Hanning-window algorithms considered here. ${ }^{1}$ In particular, it would allow the 
full velocity range of the interferometer (i.e., between 0 and the Nyquist velocity limit) to be utilized. Under such circumstances, criterion 2 would no longer be applicable and criterion 1 could then become the most appropriate of the three for predicting the success rate of the phase unwrapping.

\subsection{Vibration-induced phase errors}

If several PS algorithms are able to track the phase, i.e. the framing rate and the window duration have been set so the unwrapping success rate is sufficiently close to 1 , it is desirable to select the method that minimizes the rms phase change error produced by the vibration. As an example, Table 3 shows the average rms phase error $\bar{\sigma}$ calculated according to Eqn. (16) for different PS algorithms over a range of framing rates and $\sigma_{\phi}=\pi$, from the simulated intensity data. The corresponding unwrapping success rate for all the cases is greater than 0.95 (see Table 1). Each $\bar{\sigma}$ value was obtained using a single 2.5 second random signal with $s_{j}=\mathrm{s} / 5$ starting points. These results may be interpreted as minimum values that could be obtained for optimal experimental working conditions, i.e. without the presence of other noise sources (intensity noise, non-linearity of the camera or the phase shifter device or speckle decorrelation).

For the Carré algorithm it is seen that the rms phase error decreases as the framing rate of the camera increases. This effect does not occur for the Hanning window algorithms, for which the average rms phase error is almost independent of framing rate. The Hanning window duration does, however, have a significant effect on the average phase error: each factor of 2 increase in window size produces a reduction in the phase error by a factor of $\sim 8 \times$. By contrast, the phase error due to random errors in the measured intensity scales approximately as $1 / \sqrt{ } \mathrm{M},{ }^{19}$ and that due to speckle decorrelation is almost independent of $\mathrm{M}$. This result is interesting, 
therefore, because it suggests that under conditions of low vibration intensity the effect of the vibration can be reduced below the level of the other noise sources by increasing the duration of the time window. Experimentally-derived values are presented in Table 4. In view of the problems with unwrapping reliably the high amplitude vibrations, we include only the amplitudes of $\lambda / 4$ and below. These results indicate that the duration of the Hanning window has nearly no effect on the average phase error. As stated above, this may be related to other error sources such as intensity and decorrelation errors, which are present in the experiment but not in the simulation.

\section{Conclusions}

A controlled experiment has been designed to measure the rms phase error that appears when a high-speed speckle interferometer is used together with different PS algorithms in the presence of piston random vibrations. Due to the complexity of the experiment and the limited range of the vibration rms amplitude that could be generated, a numerical simulation has also been performed. It is shown that more important than the rms phase error is the ability to track the phase during the time history of the signal. A measure of this ability is obtained by evaluating the unwrapping success rate for different PS algorithms. It is seen that the unwrapping success rate depends mainly on the duration of the PS algorithm window, with short window algorithms offering greater unwrapping reliability, and also on the rms displacement amplitude of the vibration. Three criteria for predicting the probability of unwrapping success were investigated. The Nyquist velocity limit was found not to be the direct controlling factor. Instead, a criterion for splitting of the signal peak, based on the spectral content lying above the characteristic frequency of the PS window, was found to agree better with the simulation results. 
Provided that the unwrapping reliability is close to unity, one can then choose the PS algorithm which gives the minimum average rms phase error in order to obtain unwrapped phase maps with the highest signal-to-noise ratio. The Carré algorithm proved to combine a high unwrapping success rate together with quite a small average rms phase error for rms displacement amplitudes below $3 \lambda / 8$. For Hanning window algorithms, as the window width $M$ increases, the average rms phase error due to vibration decreases much faster than that due to other error sources, but with progressively lower chances of a successful unwrapping.

\section{Acknowledgements}

The authors wish to thank Fundación Antorchas of Argentina and The British Council for the research award to support P. D. Ruiz's visit to Loughborough University, and to the Engineering and Physical Sciences Research Council for funding under contract GR/M57835. 


\section{References}

1. X. C. de Lega, "Processing of non-stationary interference patterns: adapted phaseshifting algorithms and wavelet analysis. Application to dynamic deformation measurements by holographic and speckle interferometry", Ph.D. Dissertation, EPFL, Lausanne (1997).

2. J. M. Huntley, G. H. Kaufmann, and D. Kerr, "Phase-shifted dynamic speckle pattern interferometry at 1 kHz", Appl. Opt. 38, 6556-6563 (1999).

3. P. Haible, M. P. Kothiyal, and H. J. Tiziani, "Heterodyne temporal specklepattern interferometry", Appl. Opt. 39, 114-117 (2000).

4. J. M. Kilpatrick, A. J. Moore, J. S. Barton, J. D. C. Jones, M. Reeves, and C. Buckberry, "Measurement of complex surface deformation at audio acoustic frequencies by high-speed dynamic phase-stepped ESPI”, Opt. Lett. (2000).

5. J. M. Huntley and H. Saldner, "Temporal phase-unwrapping algorithm for automated interferogram analysis", Appl. Opt. 32, 3047-3052 (1993).

6. See, for example, D. W. Robinson and G. T. Reid, eds., Interferogram Analysis (Institute of Physics, Bristol, 1993).

7. J. Schwider, R. Burow, K. E. Elssner, J. Grzanna, R. Spolaczyk, and K. Merkel, "Digital wave-front measuring interferometry - some systematic error sources", Appl. Opt. 22, 3421-3432 (1983).

8. P. de Groot, "Vibration in phase-shifting interferometry", J. Opt. Soc. Am. A 12, 354-365 (1995).

9. P. de Groot and L. L. Deck, "Numerical simulations of vibration in phase-shifting interferometry", Appl. Opt. 35, 2172-2178 (1996). 
10. P. D. Ruiz, J. M. Huntley, J. Shen, C. R. Coggrave and G. H. Kaufmann, "Vibration-induced phase errors in high-speed phase-shifting speckle pattern interferometry", Appl. Opt. (in press).

11. B. Bessason, C. Madshus, H. A. Frøystein and H. Kolbjørnsen, "Vibration criteria for metrology laboratories”, Meas. Sci. Technol. 10, 1009-1014 (1999). 12. E. E. Ungar, D. H. Sturz and C. H. Amick, "Vibration Control Design of High Technology Facilities", Sound and Vibration, 7, 20-27 (1990).

13. Y. Surrel, "Additive noise effect in digital phase detection", Appl. Opt. 36, 271-276 (1997).

14. C. P. Brophy, "Effect of intensity error correlation on the computed phase of phase-shifting interferometry”, J. Opt. Soc. Am. A 7, 537-541 (1990).

15. P. de Groot, "Derivation of algorithms for phase-shifting interferometry using the concept of a data-sampling window. ", Appl. Opt. 34, 4723-4730 (1995).

16. Y. Surrel, "Design of algorithms for phase measurements by the use of phasestepping", Appl. Opt. 35, 51-60 (1996).

17. J. M. Huntley and H. O. Saldner, "Error-reduction methods for shape measurement by temporal phase unwrapping”, J. Opt. Soc. Am. A. 14, 3188-3196 (1997).

18. J. D. Robson, "An introduction to random vibration", Edinburgh University Press (1963).

19. Y. Surrel, "Additive noise effect in digital phase detection", Appl. Opt. 36, 271276 (1997). 


\section{Figure Captions}

Figure 1. Dynamic high-speed phase-shifting speckle interferometer showing: frame store $(\mathrm{F})$, Pockels cell $(\mathrm{P})$, high voltage driver (D), function generator (G), digital to analog converter (DA), signal conditioner (SC), high voltage amplifier (HA), test object (O), 90:10 beam splitters (BS), mirrors (M) and lenses (L).

Figure 2. Velocity spectrum of the random vibration used to excite the PZT transducer for a $\lambda / 2 \mathrm{rms}$ amplitude of the disk displacement.

Figure 3. Detail of the unwrapped phase-change measured at different locations of a disk submitted to piston random vibration for a rms displacement amplitude of $\sigma_{z}=\lambda / 4$. Each curve corresponds to the phase of pixel clusters with different starting phase. The mean phase over all the clusters is also shown in bold.

Figure 4. (a) Velocity of a surface excited with a random vibration with a rms phase amplitude of $\pi / 2$ radians. (b) Modulus, in arbitrary units, calculated from the windowed Fourier transform of the intensity signal using a Hanning window with $M=32$. (c) Idem for $M=64$. The framing rate of the camera corresponds to 1 $\mathrm{kHz}$.

Figure 5. (a) Velocity of a surface excited with a random vibration with rms phase amplitude of $2 \pi$ radians. (b) Modulus, in arbitrary units, calculated from the windowed Fourier transform of the intensity signal using a Hanning window with $M=32$. (c) Idem for $M=64$. The framing rate of the camera corresponds to $1 \mathrm{kHz}$. 
Table 2. Comparison of simulation and analytical (using criterion 3) unwrapping success rates for a range of vibration amplitudes $\left(\sigma_{\phi}\right)$ and Hanning window durations $(M)$.

\begin{tabular}{|c|c|c|c|c|c|}
\hline$\sigma_{\phi}(\mathrm{rad})$ & $\begin{array}{l}\text { Simulation (S) or } \\
\text { analytical (A) }\end{array}$ & $M=8$ & $M=16$ & $\mathrm{M}=32$ & $M=64$ \\
\hline \multirow[t]{2}{*}{$5 \pi / 2$} & $\mathrm{~S}$ & 0.65 & 0.29 & 0.22 & 0.17 \\
\hline & $\mathrm{A}$ & 0.40 & 0.00 & 0.00 & 0.00 \\
\hline \multirow[t]{2}{*}{$2 \pi$} & $S$ & 0.93 & 0.39 & 0.22 & 0.19 \\
\hline & $\mathrm{A}$ & 0.98 & 0.18 & 0.00 & 0.00 \\
\hline \multirow[t]{2}{*}{$3 \pi / 2$} & $S$ & 0.99 & 0.63 & 0.32 & 0.28 \\
\hline & $\mathrm{A}$ & 1.00 & 0.98 & 0.70 & 0.24 \\
\hline \multirow[t]{2}{*}{$\pi$} & $S$ & 1.00 & 0.98 & 0.75 & 0.44 \\
\hline & $\mathrm{A}$ & 1.00 & 1.00 & 1.00 & 1.00 \\
\hline \multirow[t]{2}{*}{$\pi / 2$} & $S$ & 1.00 & 1.00 & 1.00 & 0.98 \\
\hline & A & 1.00 & 1.00 & 1.00 & 1.00 \\
\hline
\end{tabular}


Table 3. Average rms phase error (in radians) obtained for different PS algorithms using simulated data at different framing rates. Values for cases with a success rate lower than 0.95 were not calculated (see Table $1, \sigma_{\phi}=\pi$ ).

\begin{tabular}{|c|c|c|c|c|c|c|c|}
\hline \multirow{2}{*}{$\sigma_{\phi}(\mathrm{rad})$} & \multirow{2}{*}{$\sigma_{\mathrm{z}}(\mu \mathrm{m})$} & \multirow{2}{*}{$\begin{array}{c}\text { Framing } \\
\text { rate } \\
(\mathrm{kHz})\end{array}$} & \multirow{2}{*}{ Carré } & \multicolumn{4}{|c|}{ Hanning } \\
\hline & & & & $M=8$ & $M=16$ & $M=32$ & $\mathrm{M}=64$ \\
\hline \multirow{3}{*}{$\pi$} & & 1 & 0.013640 & 0.022154 & 0.003510 & --- & --- \\
\hline & $\lambda / 4$ & 2 & 0.004176 & 0.021078 & 0.002716 & 0.000363 & --- \\
\hline & & 4 & 0.001116 & 0.020778 & 0.002625 & 0.000339 & 0.000045 \\
\hline
\end{tabular}


Table 4. Rms phase error averaged over different pixel clusters and 500 starting points (reference frames) evaluated from experimental data for different PS algorithms and vibration amplitudes. The framing rate of the system was $1 \mathrm{kHz}$.

\begin{tabular}{ccccccc}
\hline$\sigma_{\phi}$ & $\sigma_{z}$ & Carré & Hanning8 & Hanning16 & Hanning32 & Hanning64 \\
\hline $2 \pi$ & $\lambda / 2$ & 0.0949 & 0.1747 & 0.0808 & -- & -- \\
$\pi$ & $\lambda / 4$ & 0.0873 & 0.1486 & 0.0732 & -- & -- \\
$\pi / 2$ & $\lambda / 8$ & 0.0809 & 0.2325 & 0.0710 & 0.0681 & 0.0657 \\
$\pi / 4$ & $\lambda / 16$ & 0.0596 & 0.3077 & 0.0430 & 0.0375 & 0.0372 \\
$\pi / 8$ & $\lambda / 32$ & 0.0738 & 0.1320 & 0.0645 & 0.0630 & 0.0621 \\
\hline
\end{tabular}




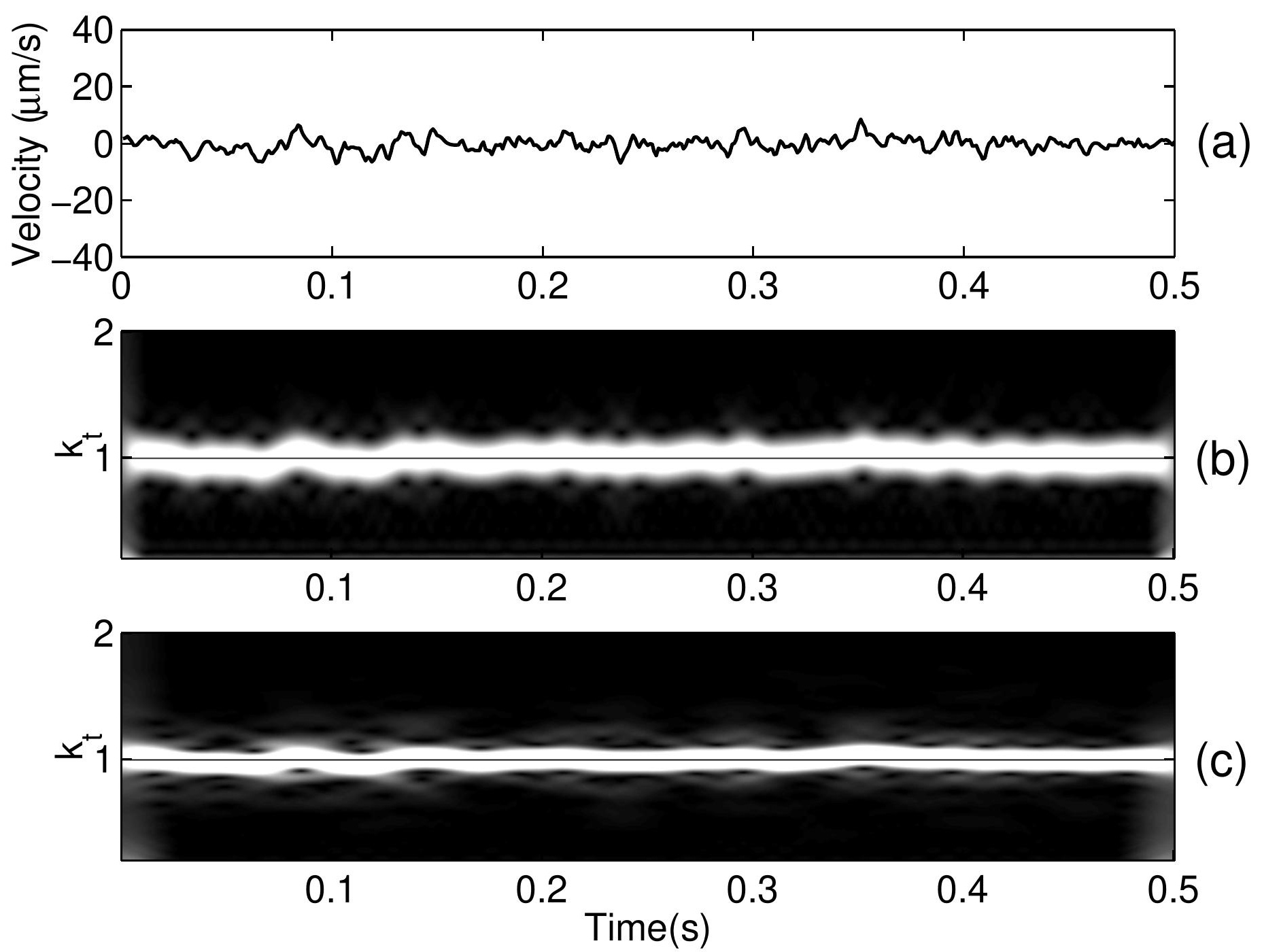




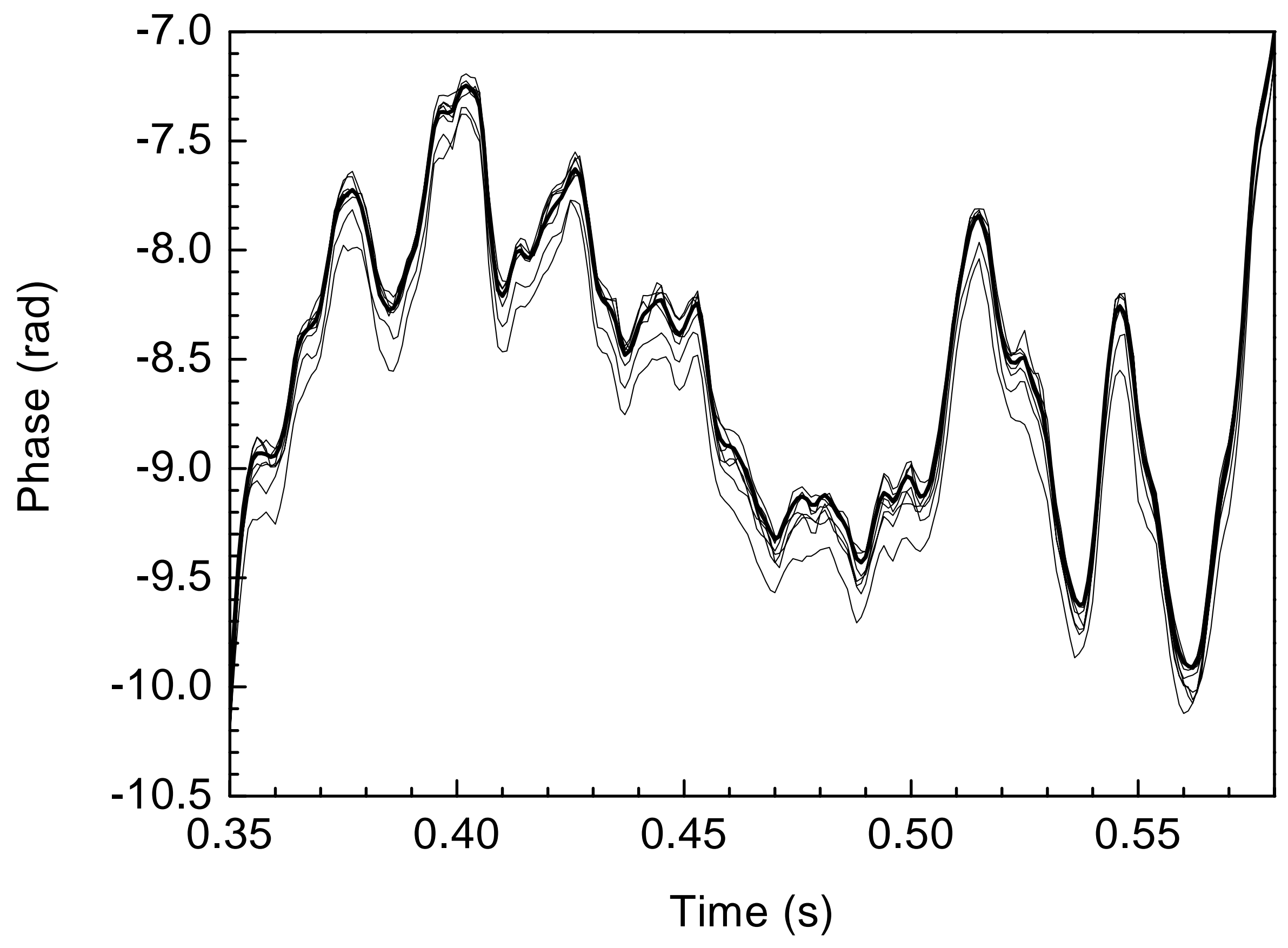


Velocity power spectral density $\left(\mu \mathrm{m}^{2} / \mathrm{s}\right)$

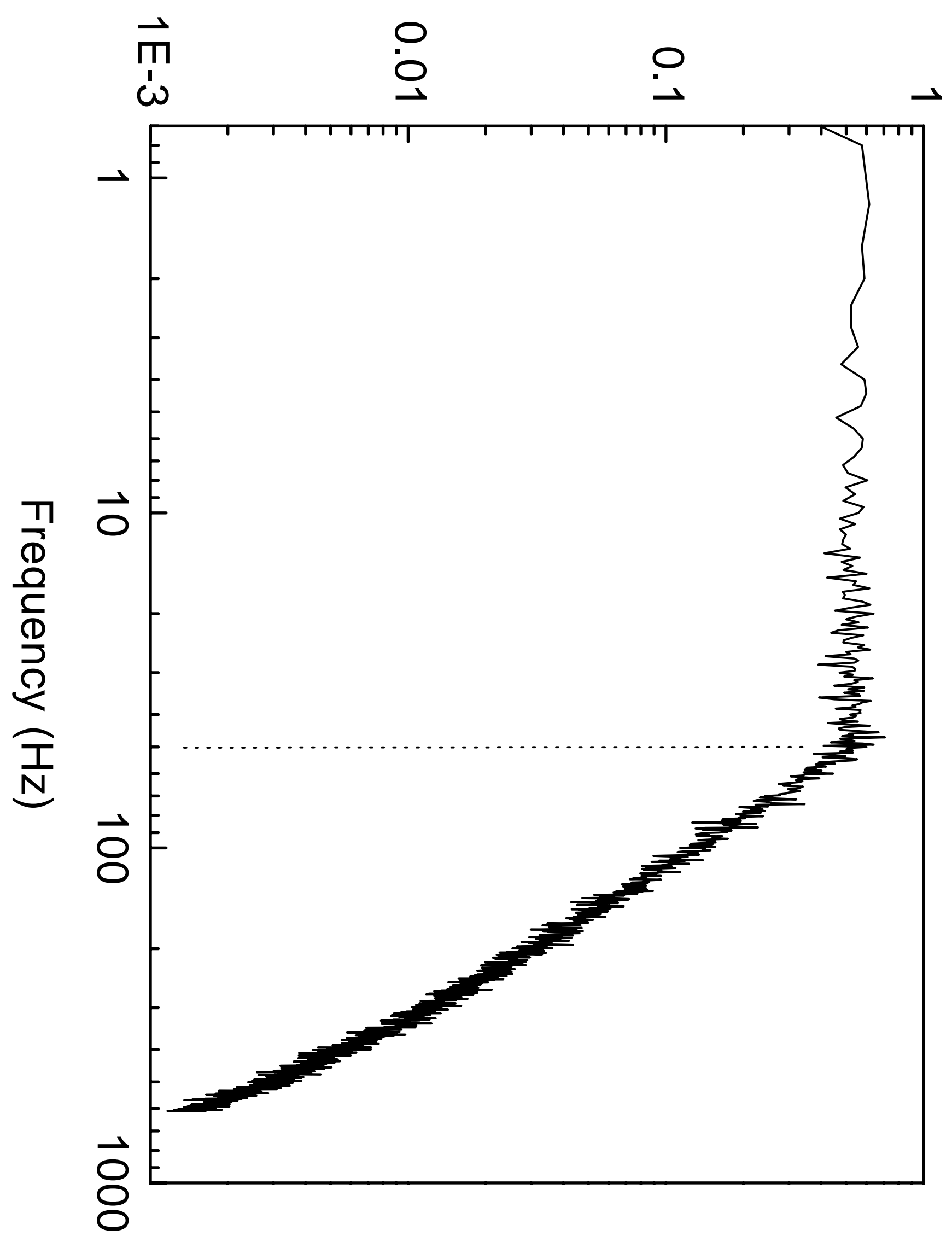




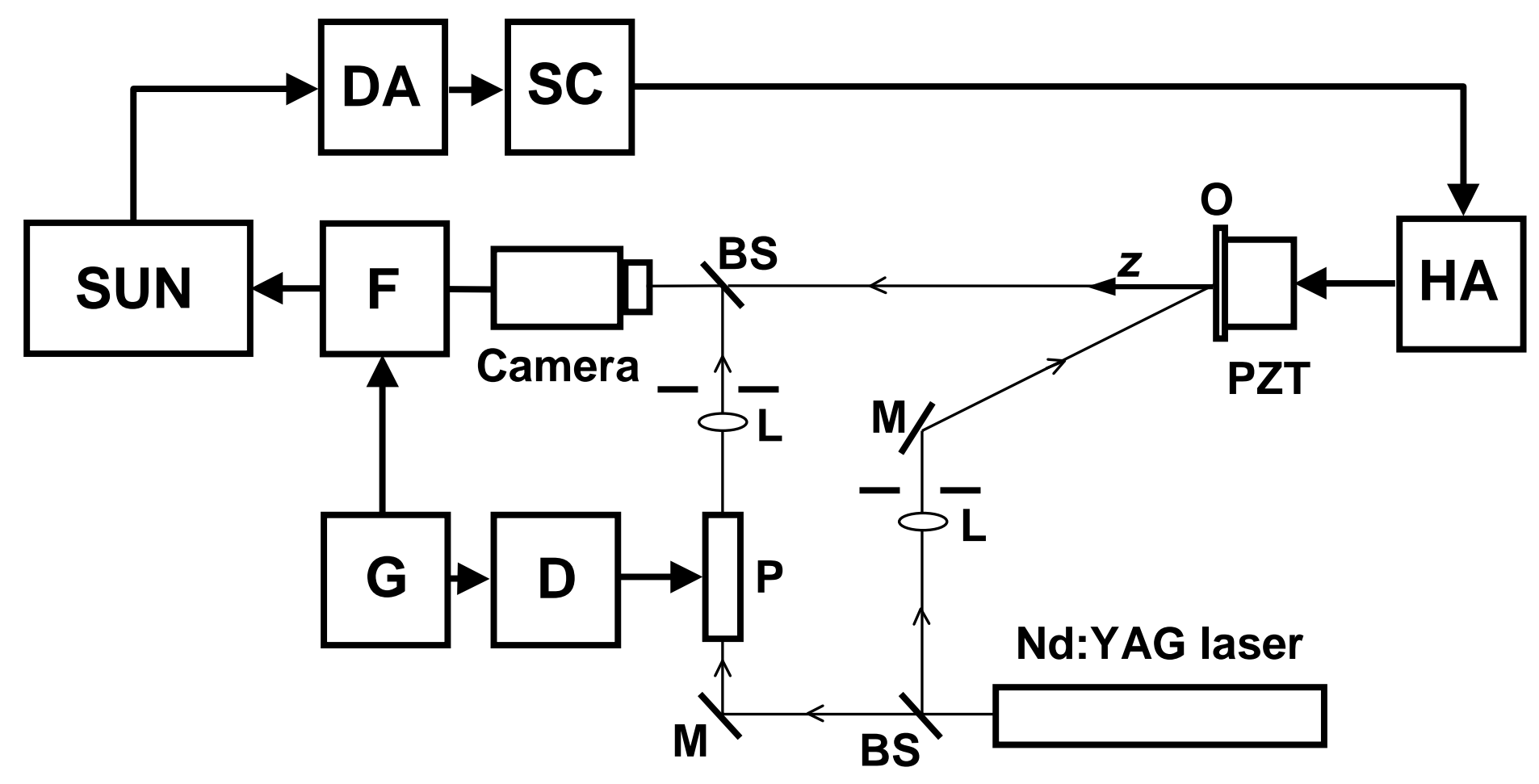



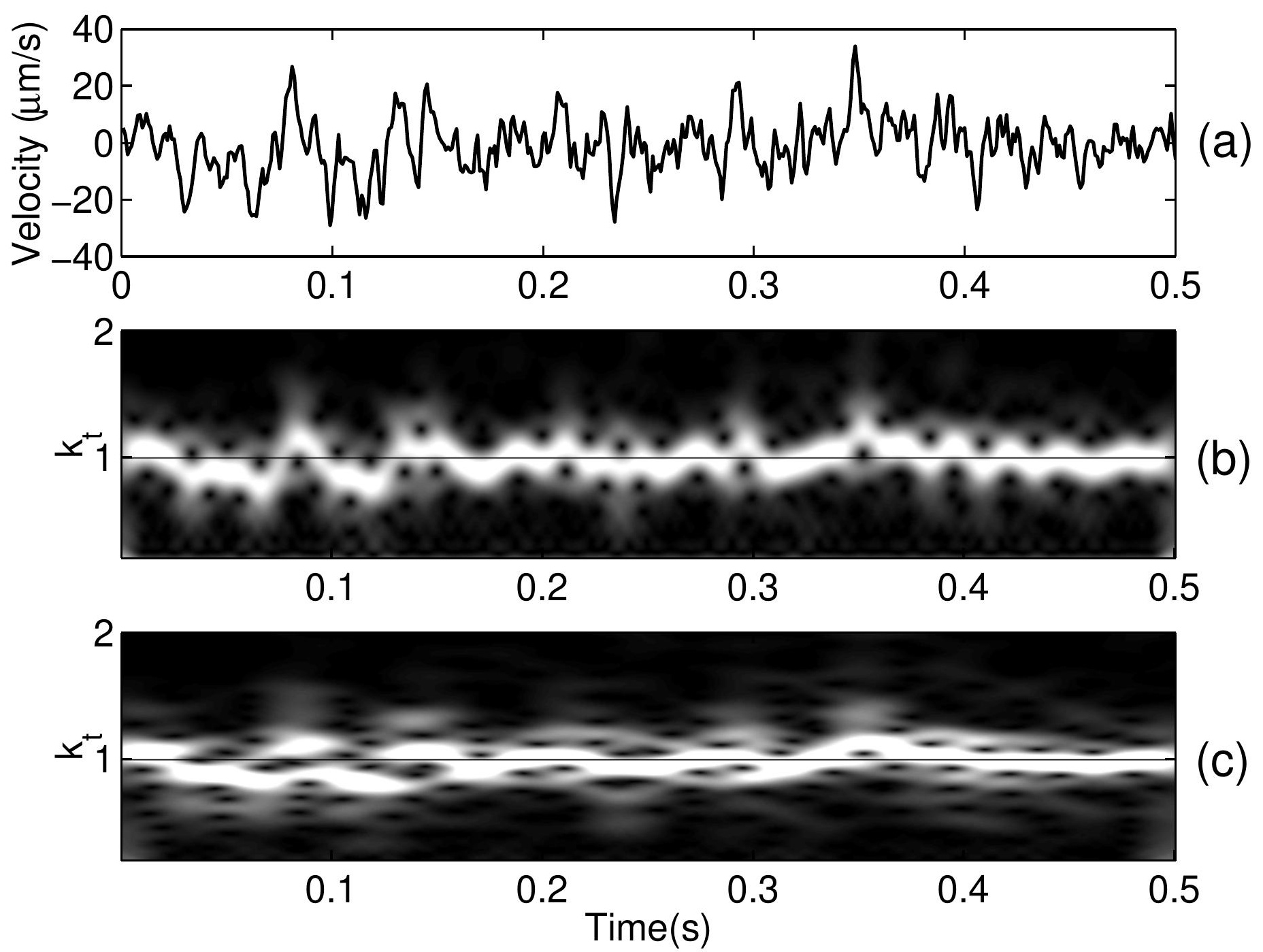

(c)

(b)

Time(s) 